\title{
Resolving the wave vector in negative refractive index media
}

\author{
S. Anantha Ramakrishna \\ Department of Physics, Indian Institute of Technology, Kanpur - 208 016, India \\ Olivier J. F. Martin \\ Nanophotonics and Metrology Laboratory, Swiss Federal Institute of Technology Lausanne, \\ EPFL-STI-NAM, 1015 Lausanne, Switzerland
}

\begin{abstract}
Received April 7, 2005; revised manuscript received June 9, 2005; accepted June 10, 2005
We address the general issue of resolving the wave vector in complex electromagnetic media including negative refractive media. This requires us to make a physical choice of the sign of a square root imposed merely by conditions of causality. By considering the analytic behavior of the wave vector in the complex plane, it is shown that there are a total of eight physically distinct cases in the four quadrants of two Riemann sheets. (C) 2005 Optical Society of America

OCIS codes: $000.2690,260.2110,350.5500$.
\end{abstract}

The electromagnetic response of a homogeneous medium is usually charecterized by the dielectric constant $(\epsilon)$ and the magnetic permeability $(\mu)$. The refractive index $(n)$ first arises in the context of the wave equation and is defined by $n^{2}=\epsilon \mu$. For most optical media, $\mu=1$ and $n$ is taken to be $\sqrt{\epsilon}$. These are in general complex functions (i.e., $\epsilon=\epsilon^{\prime}+\mathrm{i} \epsilon^{\prime \prime}$ and $\mu=\mu^{\prime}$ $\left.+\mathrm{i} \mu^{\prime \prime}\right)$ of the frequency $(\omega)$ of the applied radiation. For media at thermodynamic equilibrium, it is usually demanded that the imaginary parts of $\epsilon(\omega)$ and $\mu(\omega)$ be positive so that the total energy dissipated by the electromagnetic fields in a volume $(V)$,

$$
\int_{V} \mathrm{~d}^{3} r \int_{-\infty}^{\infty} \omega\left[\epsilon^{\prime \prime}(\omega)|\mathbf{E}(\mathbf{r}, \omega)|^{2}+\mu^{\prime \prime}(\omega)|\mathbf{H}(\mathbf{r}, \omega)|^{2}\right] \frac{\mathrm{d} \omega}{2 \pi},
$$

is positive. ${ }^{1}$ However, the signs of the real parts of $\epsilon$ and $\mu$ are not subject to any such restriction. ${ }^{1}$ Veselago ${ }^{2}$ concluded that a material with real and simultaneously negative $\epsilon$ and $\mu$ at a given frequency would have a negative refractive index $n=-\sqrt{\epsilon \mu}$. This result remained an academic curiosity until recently, when it became possible to fabricate structured metamaterials with negative $\epsilon$ and $\mu .^{3-7}$ The possibility that a negative refractive index may open a door to perfect lenses ${ }^{8}$ that are not subject to the diffraction limit has given a great impetus to the study of these materials.

The sign of the refractive index in these media has been the subject of some debate. Smith and Kroll analyzed the problem of a current sheet radiating into a medium with negative $\epsilon$ and negative $\mu$ and concluded that $n<0$ for power to flow away from the source. This has been criticized by some authors ${ }^{10,11}$ and supported by others. ${ }^{12}$ However, there exists a real problem of choosing the sign of the square root to determine the wave vector of a wave transmitted into a negative medium. For the case of normally incident propagating waves with no tranverse components, this sign of the wave vector directly corresponds to the sign of the refractive index.

Here we consider this problem of choosing the correct wave vector in media with complex $\epsilon$ and $\mu$. Using the theory of analytic functions we show that there is, indeed, a physical choice of the sign to be made. We include the cases of propagating and evanescent waves and that where both the real and the imaginary parts of $\epsilon$ and $\mu$ can be positive or negative. Negative imaginary parts are possible in an amplifying medium (as in a laser). Even for the case of passive metamaterials that are overall only dissipative, it appears possible to have a negative imaginary part of $\epsilon$ when the real part of $\mu$ is negative ${ }^{13}$ and a negative imaginary part of $\mu$ when the real part of $\epsilon$ is negative. ${ }^{14}$ We show that there are a total of eight distinct physical cases in two Riemann sheets for the square root operation. Our results support the case that when a medium has predominantly real and simultaneously negative $\epsilon$ and $\mu$ at a given frequency, it must be considered to have a negative refractive index.

Let us examine the choice of the wave vector in homogeneous media. The propagation of light in a medium is governed by Maxwell's equations. For a timeharmonic plane wave, $\exp [i(\mathbf{k} \cdot \mathbf{r}-\omega t)]$, with an angular frequency $\omega$ and a wave vector $\mathbf{k}$, these reduce to

$$
\mathbf{k} \times \mathbf{E}=\frac{\omega}{\mathrm{c}} \mu \mathbf{H}, \quad k \times H=-\frac{\omega}{\mathrm{c}} \epsilon \mathbf{E},
$$

where $\mathbf{E}$ and $\mathbf{H}$ are the electric and magnetic fields associated with the wave, respectively. For complex $\epsilon$, $\mu$, and $\mathbf{k}$, the wave becomes inhomogeneous. Writing $\epsilon=\epsilon^{\prime}+i \epsilon^{\prime \prime}$, and $\mu=\mu^{\prime}+i \mu^{\prime \prime}$, we note that the medium is absorbing if $\epsilon^{\prime \prime}>0, \mu^{\prime \prime}>0$, and amplifying if $\epsilon^{\prime \prime}<0$, $\mu^{\prime \prime}<0$. The mechanism for the absorption or amplification, of course, lies in the underlying electric or magnetic polarizabilities. We should mention here that a metamaterial can exhibit resonances unrelated to the underlying material polarization, for ex- 
ample, an LC resonance for split-ring resonators., ${ }^{3,13}$ But these resonances can also be subsumed into an effective macroscopic $\epsilon$ and $\mu$ when the wavelength of the radiation is much larger than the underlying structure. Then the structure appears homogeneous. In an isotropic medium, Maxwell's equations require that

$$
|\mathbf{k}|^{2}=k_{x}^{2}+k_{y}^{2}+k_{z}^{2}=\epsilon \mu \frac{\omega^{2}}{c^{2}}
$$

which describes the dispersion in the medium. To determine the wave vector $\mathbf{k}$ in the medium, we have to carry out a square root operation. Obviously the choice of the sign of the square root will have to be made consistent with the Maxwell's equations and causality.

Without loss of generality, we consider an electromagnetic wave with a wave vector $\left[k_{x}, 0, k_{z}\right]$ to be incident from vacuum on the left $(-\infty<z<0)$ on a semi-infinite medium $(\infty>z>0)$ with arbitrary values of $\epsilon$ and $\mu$. Due to $x$ invariance, $k_{x}$ is preserved across the interface. The $z$ component of the wave vector, $k_{z}$, however, has to be obtained from the dispersion relation

$$
k_{z}= \pm \sqrt{\epsilon \mu \frac{\omega^{2}}{c^{2}}-k_{x}^{2}},
$$

where a physical choice of the sign of the square root has to be made. Now the waves in medium 2 could be propagating $\left[k_{x}^{2}<\operatorname{Re}\left(\epsilon \mu \omega^{2} / c^{2}\right)\right]$ or evanescent $\left[k_{x}^{2}\right.$ $\left.>\operatorname{Re}\left(\epsilon \mu \omega^{2} / c^{2}\right)\right]$. Further, the media could be absorbing or amplifying, depending on the sign of $\operatorname{Im}(\epsilon \mu)$ in Eq. (4). This enables us to divide the complex plane for $Z=k_{z}^{2}$ into the four quadrants shown in Fig. 1. The waves corresponding to quadrants 1 and 4 have a propagating nature, and the waves corresponding to quadrants 2 and 3 are evanescent. Crucially, we note that there is a branch cut in the complex plane for $\sqrt{Z}=k_{z}$, and one cannot analytically continue the behavior of the waves across this branch cut. This branch cut divides the Riemann surface into two sheets in which the two different signs for the square root will have to be taken. ${ }^{15}$ The different regions in the $Z=k_{z}^{2}$ plane are mapped into the different physical regions of the $\sqrt{Z}$ plane depending on $k_{x}, \epsilon$, and $\mu$. For absorbing media, the wave amplitude at the infinities obviously has to disappear. For amplifying media, one has to be more careful. The only conditions are that evanescently decaying waves remain decaying, propagating ones remain propagating, and no information can flow in from the infinities. This ensures that the near-field features of a source cannot be probed at large distances merely by embedding the source in an amplifying medium. Due to the above reasons we will choose the branch cut along the negative imaginary axis as shown in Fig. 1. This proves to be a convenient choice. This choice corresponds to our conventional listing of the different media as we move around the complex $k_{z}^{2}$ plane (Fig. 1) in the counterclockwise direction. Hence our range

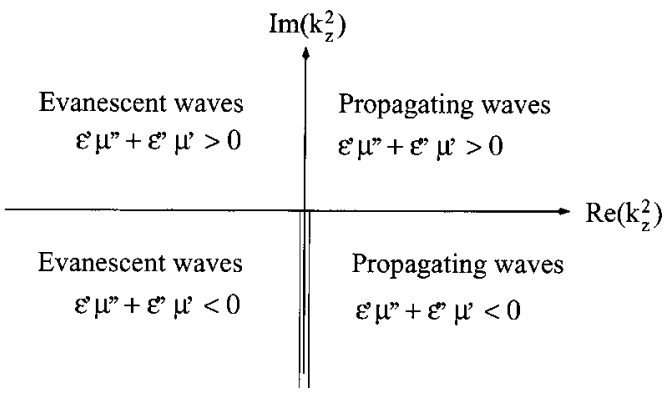

Fig. 1. Complex plane for $k_{z}^{2}$, showing the different regions for the propagating and evanescent waves. The branch cut along the negative imaginary axis for the square root is shown.

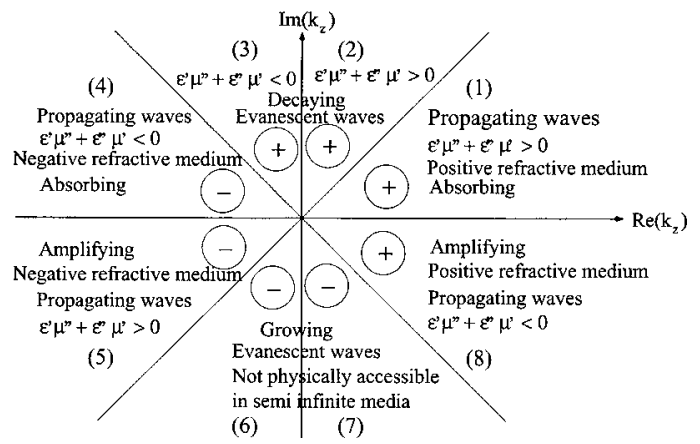

Fig. 2. Complex plane for $k_{z}=\sqrt{Z}$ with the corresponding eight regions in the two Riemann sheets for $k_{z}^{2}$. The evanescent regions could be absorbing or amplifying as explained in the text, depending on the signs of $\epsilon^{\prime}$ and $\mu^{\prime}$. The sign of the square root for each region is shown enclosed in a small circle.

for the argument $\theta$ of $k_{z}^{2}$ becomes $-\pi / 2<\theta<3 \pi / 2$ for the first Riemann sheet and $3 \pi / 2<\theta<7 \pi / 2$ for the second Riemann sheet, corresponding to the two signs of the square root $k_{z}= \pm \sqrt{Z}=|Z|^{1 / 2} e^{i \theta / 2}$ and $|Z|^{1 / 2} e^{i \pi+i \theta / 2}$. The complex plane for $k_{z}=\sqrt{Z}$ with the corresponding eight regions in the two Riemann sheets is shown in Fig. 2. We now consider the behavior of the waves individually in each of these regions.

Region 1: $0<\operatorname{Arg}\left(k_{z}\right)<\pi / 4$ corresponding to 0 $<\operatorname{Arg}\left(k_{z}^{2}\right)<\pi / 2$. This is the conventional case of a propagating wave in a positively refracting absorbing medium. Here $\mu^{\prime} \epsilon^{\prime \prime}+\epsilon^{\prime} \mu^{\prime \prime}>0$ implying absorption. The wave decays in amplitude as it propagates in the medium. We also have $\epsilon^{\prime}>0$ and $\mu^{\prime}>0$ and choose the positive sign of the square root.

Region 2: $\pi / 4<\operatorname{Arg}\left(k_{z}\right)<\pi / 2$ corresponding to $\pi / 2<\operatorname{Arg}\left(k_{z}^{2}\right)<\pi$. This is a case of evanescently decaying waves. We have $\epsilon^{\prime} \mu^{\prime \prime}+\epsilon^{\prime \prime} \mu^{\prime}>0$, which implies overall absorption, for example, $\left(\epsilon^{\prime \prime}>0, \mu^{\prime \prime}>0\right)$ if $\epsilon^{\prime}$ $>0, \mu^{\prime}>0$ (positive refractive index) or amplification $\left(\epsilon^{\prime \prime}<0, \mu^{\prime \prime}<0\right)$ if $\epsilon^{\prime}<0, \mu^{\prime}<0$ (negative refractive index). Note that it is the overall sign of $\epsilon^{\prime} \mu^{\prime \prime}+\epsilon^{\prime \prime} \mu^{\prime}$ that matters. ${ }^{16}$ In either case we have an evanescent wave that decays into the medium to zero as $\mathrm{z} \rightarrow \infty$.

Region 3: $\pi / 2<\operatorname{Arg}\left(k_{z}\right)<3 \pi / 4$ corresponding to $\pi$ $<\operatorname{Arg}\left(k_{z}^{2}\right)<3 \pi / 2$. This is similiar to region 2 and corresponds to evanescently decaying waves, although we now have $\epsilon^{\prime} \mu^{\prime \prime}+\epsilon^{\prime \prime} \mu^{\prime}<0$, implying absorption for 
negative refractive media and amplification for positive refractive media. Again we choose the positive square root, and we have only evanescently decaying waves in the semi-infinite medium.

Region 4: $3 \pi / 4<\operatorname{Arg}\left(k_{z}\right)<\pi$ corresponding to $3 \pi / 2<\operatorname{Arg}\left(k_{z}^{2}\right)<2 \pi$. Now we move into the second Riemann sheet and choose the negative sign for the square root. This region corresponds to negatively refracting media $\left(\epsilon^{\prime}<0, \mu^{\prime}<0\right)$ and absorbing media $\left(\epsilon^{\prime} \mu^{\prime \prime}+\epsilon^{\prime \prime} \mu^{\prime}<0\right)$ (Ref. 16) and hence, for example, $\epsilon^{\prime \prime}$ $>0, \mu^{\prime \prime}>0$. We have propagating waves (albeit lefthanded) that decay in amplitude as the wave propagates into the medium. Note that the negative sign is crucial to ensure this decaying nature of the waves in absorbing media.

Region 5. $\pi<\operatorname{Arg}\left(k_{z}\right)<5 \pi / 4$ corresponding to $2 \pi$ $<\operatorname{Arg}\left(k_{z}^{2}\right)<5 \pi / 2$. We again have the negative sign for the square root and propagating waves in negative index media. But note that $\epsilon^{\prime} \mu^{\prime \prime}+\epsilon^{\prime \prime} \mu^{\prime}>0$, implying, for example, that $\epsilon^{\prime \prime}<0, \mu^{\prime \prime}<0$. In general, overall there is amplification and the waves grow exponentially with propagation distance.

Region 6. $5 \pi / 4<\operatorname{Arg}\left(k_{z}\right)<3 \pi / 2$ corresponding to $5 \pi / 2<\operatorname{Arg}\left(k_{z}^{2}\right)<3 \pi$. This corresponds to exponentially growing evanescent waves that are not physically accessible in semi-infinite media. For finite media (slabs) these solutions are permissible and are responsible for the perfect lens effect. ${ }^{8}$

Region 7. $3 \pi / 2<\operatorname{Arg}\left(k_{z}\right)<7 \pi / 4$ corresponding to $3 \pi<\operatorname{Arg}\left(k_{z}^{2}\right)<7 \pi / 2$. This also corresponds to exponentially growing evanescent waves and are not physically accessible in semi-infinite media.

Region 8. $-\pi / 4<\operatorname{Arg}\left(k_{z}\right)<0$ corresponding to $-\pi / 2<\operatorname{Arg}\left(k_{z}^{2}\right)<0$. Now we are back on the first Riemann sheet and choose the positive sign for the square root. This is the conventional case of propagating waves in amplifying positive refractive media, which grow exponentially in amplitude into the medium.

Note that in all these cases it is not $\epsilon$ or $\mu$ that individually determines the nature of the waves but a combination of them determined by $\operatorname{Re}\left(k_{z}^{2}\right)$ and $\operatorname{Im}\left(k_{z}^{2}\right)$. For evanescent waves in a semi-infinite medium, we always choose the positive square root so that $\operatorname{Im}\left(k_{z}\right) \geqslant 0$. In the case of evanescent waves in amplifying media, our choice results in a Poynting vector in the medium that points toward the source (interface in this case). This, however, does not violate causality as the Poynting vector-energy flow decays exponentially to zero at infinity and no information flows in from the infinities. This counterintuitive behavior does not imply that source has turned into a sink, rather it indicates that there would be a large (infinitely large for unsaturated linear gain) accumulation of energy density (intense local field enhancements) near a source. This behavior can also be understood in terms of the fundamental bosonic property of light: photons in the localized mode stimulate the amplifying medium to emit more photons into the same localized mode. In other words, the near-field modes of a source remain evanescent even inside an amplifying medium and do not affect the far field. Also note that $\epsilon^{\prime}$ and $\mu^{\prime}$ (as in a metal) could have opposite signs, in which case the waves are evanescent and fall into regions 2 or 3 depending on the overall sign of $\epsilon^{\prime} \mu^{\prime \prime}+\epsilon^{\prime \prime} \mu^{\prime}$. The importance of the sign of this quantity to determine the energy flow has also been noted by Depine and Lakhtakia. ${ }^{16}$

In summary, the problem of choosing a wave vector in complex media can be analyzed using the properties of analytic functions. The branch cut for the square root in the complex plane of $Z=k_{z}^{2}$ has to be chosen along the negative imaginary axis. This results in eight distinct cases for the quadrants in two Riemann sheets corresponding variously to propagating or evanescent waves in absorbing or amplifying media, with positive or negative real parts of $\epsilon$ and $\mu$. The positive sign of the square root has to be taken for the cases in one sheet and the negative sign in the other. The case of propagating waves in absorbing media with $\epsilon^{\prime}<0$ and $\mu^{\prime}<0$ lies in the second sheet, which justifies calling them negative refractive index media.

S. A. Ramakrishna's e-mail address is sar@iitk.ac.in.

\section{References}

1. L. D. Landau and E. M. Lifschitz, Electrodynamics of Continuous Media (Butterworth, 1984).

2. V. G. Veselago, Sov. Phys. Usp. 10, 509 (1968).

3. D. R. Smith, W. J. Padilla, D. C. Vier, S. C. NematNasser, and S. Schultz, Phys. Rev. Lett. 84, 4184 (2000).

4. R. A. Shelby, D. R. Smith, and S. Schultz, Science 292, 77 (2001).

5. A. Grbic and G. V. Eleftheriades, J. Appl. Phys. 92, 5930 (2002).

6. C. G. Parazzoli, R. B. Greegor, K. Li, B. E. C. Kotenbah, and M. Tanielan, Phys. Rev. Lett. 90, 107401 (2003).

7. A. A. Houck, J. B. Brock, and I. L. Chuang, Phys. Rev. Lett. 90, 137401 (2003).

8. J. B. Pendry, Phys. Rev. Lett. 85, 3966 (2000).

9. D. R. Smith and N. Kroll, Phys. Rev. Lett. 85, 2933 (2000)

10. P. M. Valanju, R. M. Walser, and A. P. Valanju, Phys. Rev. Lett. 88, 187401 (2002).

11. A. L. Pokrovsky and A. L. Efros, Solid State Commun. 124, 283 (2002).

12. J. Pacheco, T. M. Grzegorczyk, B.-I. Wu, Y. Zhang, and J. A. Kong, Phys. Rev. Lett. 89, 257401 (2002).

13. S. O'Brien and J. B. Pendry, J. Phys.: Condens. Matter 14, 4035 (2002).

14. T. Koschny, P. Markos, D. R. Smith, and C. M. Soukoulis, Phys. Rev. E 68, 065602(R) (2003).

15. R. V. Churchill, Complex Variables and Applications, 3rd ed. (McGraw-Hill, 1974).

16. R. A. Depine and A. Lakhtakia, Microwave Opt. Technol. Lett. 41, 315 (2004). 